\title{
La médecine spectacle
}

C'est un grand coup médiatique que la société de TV néerlandaise «Endemol» a frappé voici quelques semaines. Et un coup double: tout d'abord les ingénieux producteurs ont suscité un tsunami de protestations universel en annonçant un «grand spectacle du donneur» qui allait voir trois insuffisants rénaux se disputer les faveurs et le rein d'une jeune malade en phase terminale. «Il s'agit d'un jeu macabre d'une agence de médias ayant perdu tout sens de la morale»: tel fut l'un des commentaires les plus modérés émis à la veille de l'émission (Jörg-Dietrich Hoppe, président de la chambre médicale allemande, cité par le «Tagesspiegel online»).

Une fois le cauchemar terminé et le tout s'étant avéré un canular - la jeune femme soidisant gravement malade était une comédienne et aucun organe ne fut donné -, une vague d'indignation s'est rapidement propagée sous une forme inattendue: une grande partie des téléspectateurs ainsi menés en bateau, dont des hommes politiques, des médecins spécialistes de la transplantation et des éthiciens, a tiré inopinément un bilan positif de cette mise en scène. Certes, a-t-on dit en substance, les producteurs de l'émission avaient recouru à un truc plutôt corsé, voire même douteux, pour attirer l'attention sur la pénurie de donneurs d'organes, un sujet qui leur tenait à cœur. Mais le ministre néerlandais de l'information n'a de loin pas été le seul à estimer soudainement (toujours selon le «Tagesspiegel online») que ce spectacle télévisé avait su sensibiliser «intelligemment» le public à un problème grave.

Tiens, tiens... Les créateurs de Big Brother, connus pour leur engagement social, voulaient donc conscientiser le monde. Ils avaient une mission! C'est un fait que le fondateur de la chaîne BNN, qui a diffusé le show, avait lui même souffert d'une maladie rénale et en était mort. Selon différentes sources, soit il n'était jamais parvenu à obtenir un rein (cf. par ex. «T-onlineNachrichten»), soit il était décédé cinq ans après avoir subi une transplantation (cf. par ex. «News orf.at»). Mais bon, il serait mesquin d'insister sur de tels détails.

Et ce serait même une hérésie d'imaginer que le mécanisme ait pu fonctionner dans l'autre sens. Dans un tel cas de figure, les producteurs TV auraient fondé leur démarche sur des questions du genre: quelle stratégie nous permettra d'attirer un maximum d'attention, de faire grim- per le taux d'écoute, d'accroître notre valeur marchande et, accessoirement, de remplir un peu nos caisses? Se camper tout d'abord en vilains garçons pour se transformer ensuite par surprise - coup de clairon! - en blancs chevaliers au service d'une bonne cause. Qui pourrait trouver à redire à un projet visant une augmentation massive de la demande de cartes de donneurs d'organes dans divers pays? Qui songerait à critiquer une idée permettant à des experts et d'autres personnalités engagées de s'exprimer sérieusement sur un grave problème affectant la médecine? Comme on l'a dit, un procédé plutôt génial.

Une société a les programmes de télévision qu'elle mérite, pourrait-on aussi relever. La médecine en tant que spectacle: une approche qui, sous une forme plus ou moins décente, existe aussi chez nous depuis longtemps. Des malades posant tels des vedettes sur le plateau sont acclamés par le public réuni en studio: «J'ai le plaisir de vous présenter M. X, qui souffre d'une insuffisance cardiaque»... applaudissements. «Et voici Mme Y, atteinte de fibromyalgie»... applaudissements. «Et enfin M. Z., souffrant d'une dépression résistante au traitement»... Nouveaux applaudissements.

A l'ère des émissions-débats sur des thèmes du genre «A l'aide, mon pékinois est infidèle!», nous nous sommes malheureusement déjà habitués au caractère intrinsèquement mensonger de tels cadrages. L'espoir que tout compte fait, le bilan des programmes de ce type pourrait être positif malgré leur caractère plus que douteux, paraît hautement optimiste. Les comédies de ce genre sont consommées comme du divertissement instantané et engendrent rarement davantage qu'un sentiment de sympathie très passager. Si l'on diffusait chaque semaine un show sur le don d'organes, l'intérêt des téléspectateurs baisserait très rapidement, sans parler de la proportion de celles et ceux qui commandent ensuite une carte de donneur.

Ne reste donc que l'exploitation permanente du filon de la sensation, un genre de jeu que notre société joue apparemment volontiers. Quant à savoir jusqu'où cela ira, l'avenir le dira. Peut-être ne tarderons-nous pas à voir à l'écran trois désespérés fatigués de vivre, en train de jouer devant nous pour gagner un suicide assisté dans un hôtel de luxe?

Bruno Kesseli 